\title{
Gastroduodenal ulceration following liver radioembolization with yttrium-90
}

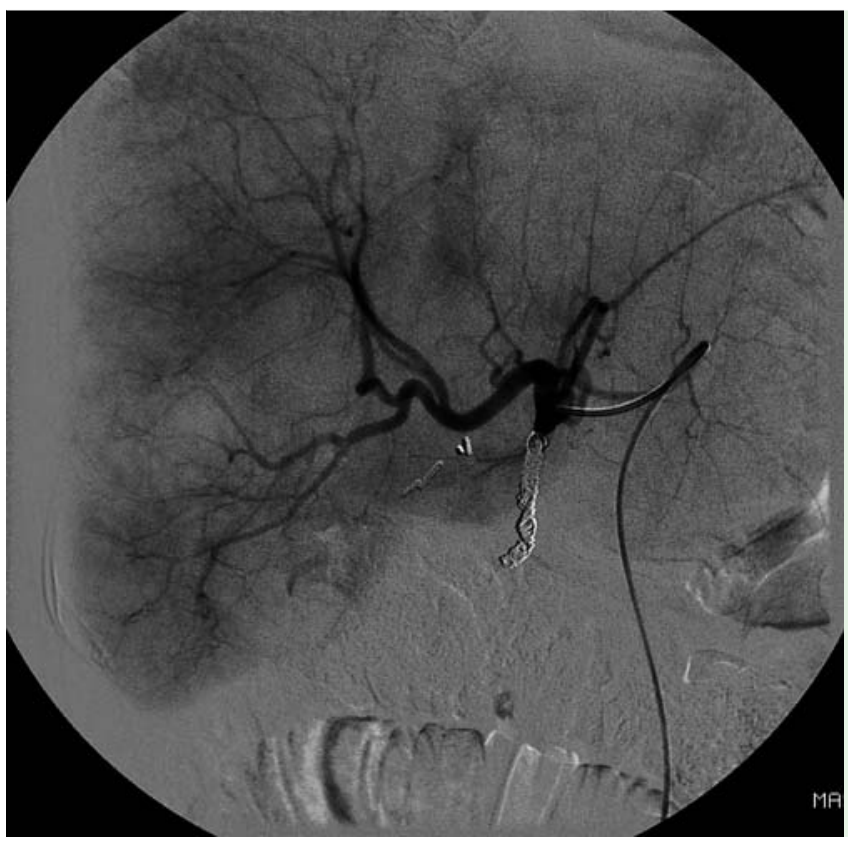

Fig. 1 Pretreatment hepatic angiogram in a 54-year-old woman with unresectable bilobar liver metastases from a rectal adenocarcinoma showing normal arterial anatomy.

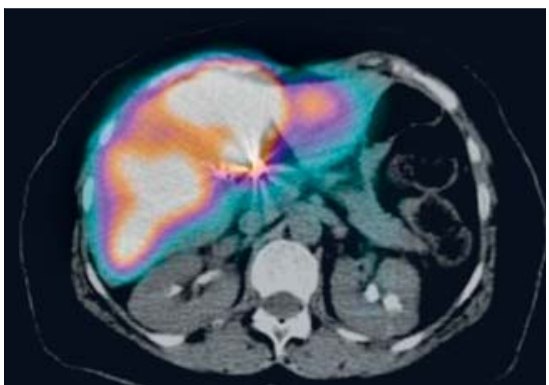

Fig. 2 Single-photon emission computed tomography (SPECT) scan after selective internal radiation therapy with yttrium-90 ( ${ }^{90} \mathrm{Y}$-SIRT) showing radioactivity in the liver only with no evidence of extrahepatic shunting into the gastrointestinal tract or lung.

Radioembolization or selective internal radiation therapy (SIRT) with yttrium-90 $\left.{ }^{90} \mathrm{Y}\right)$ resin microspheres is an effective locoregional treatment for unresectable primary and metastatic malignancies of the liver [1]. SIRT is a straightforward method of delivering microspheres containing ${ }^{90} \mathrm{Y}$ (a high-energy $\beta$-emitting radioisotope) to the microvasculature of malignancies whilst sparing the normal liver parenchyma.

A 54-year-old woman with unresectable bilobar liver metastases from a rectal adenocarcinoma was recommended to undergo ${ }^{90}$ Y-SIRT. Pretreatment hepatic an- giography revealed normal arterial anatomy ( Fig. 1). The gastroduodenal artery was embolized with eight coils to prevent nontargeted flow to the stomach and duodenum. Afterwards a ${ }^{99} \mathrm{Tc}$-macroaggregated albumin solution (to simulate the ${ }^{90} \mathrm{Y}$ microspheres) was selectively injected into the hepatic artery. This was followed by a single-photon emission computed tomography (SPECT) scan, which excluded extrahepatic shunting into the gastrointestinal tract and the lung. ${ }^{90} \mathrm{Y}$-SIRT was performed 2 weeks later. Selective catheterization of the left and right lobes of the liver permitted delivery a $1.7 \mathrm{GBq}$ dose of ${ }^{90} \mathrm{Y}$ resin microspheres. A post-treatment SPECT scan confirmed uptake in the liver only ( $\bullet$ Fig. 2).

The patient presented again having developed epigastric pain 12 weeks after undergoing the ${ }^{90}$ Y-SIRT. An upper gastrointestinal endoscopy showed a large gastric ulcer at the antrum and a superficial ulcer in the duodenal bulb ( $\bullet$ Fig.3). Biopsies revealed deep purple, round, acellular foreign bodies, compatible with ${ }^{90} \mathrm{Y}$ resin microspheres ( Fig.4). Esomeprazole (40 mg twice daily) was started and produced a gradual clinical improvement. Radioembolization-associated gastroduodenal ulceration results from nontargeted flow of microspheres through inter-relat-

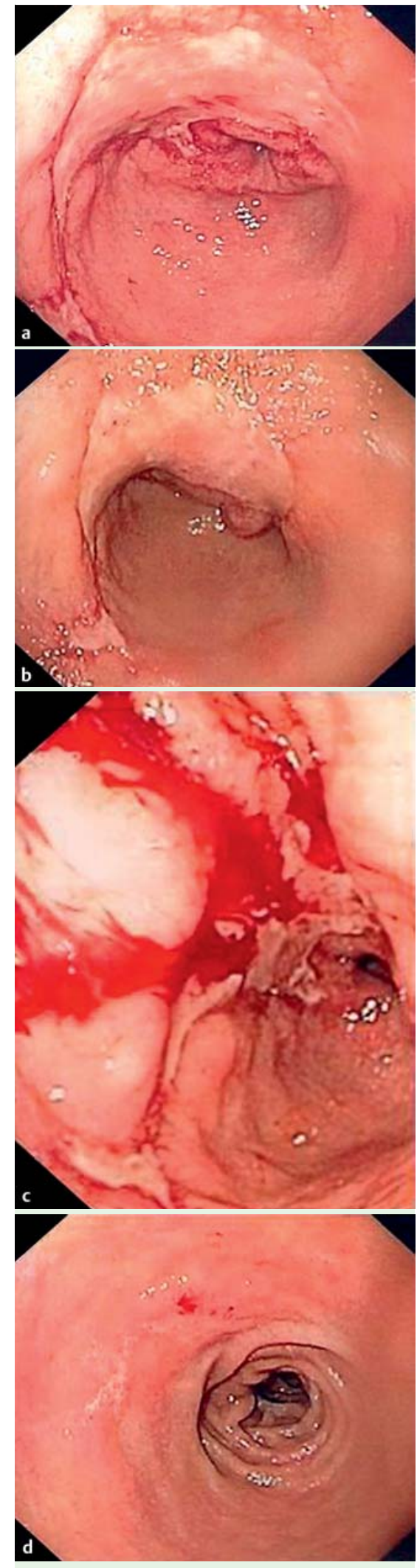

Fig.3 Views during an upper gastrointestinal endoscopy performed 12 weeks after selective internal radiation therapy (SIRT) showing: a, b a large gastric ulcer with a clean base, regular borders and whitish exudate at the lesser curvature of the antrum and marked erythema in the prepyloric region; c appearance after biopsies were taken; $\mathbf{d}$ a superficial ulcer in the anterior wall of duodenal bulb. 


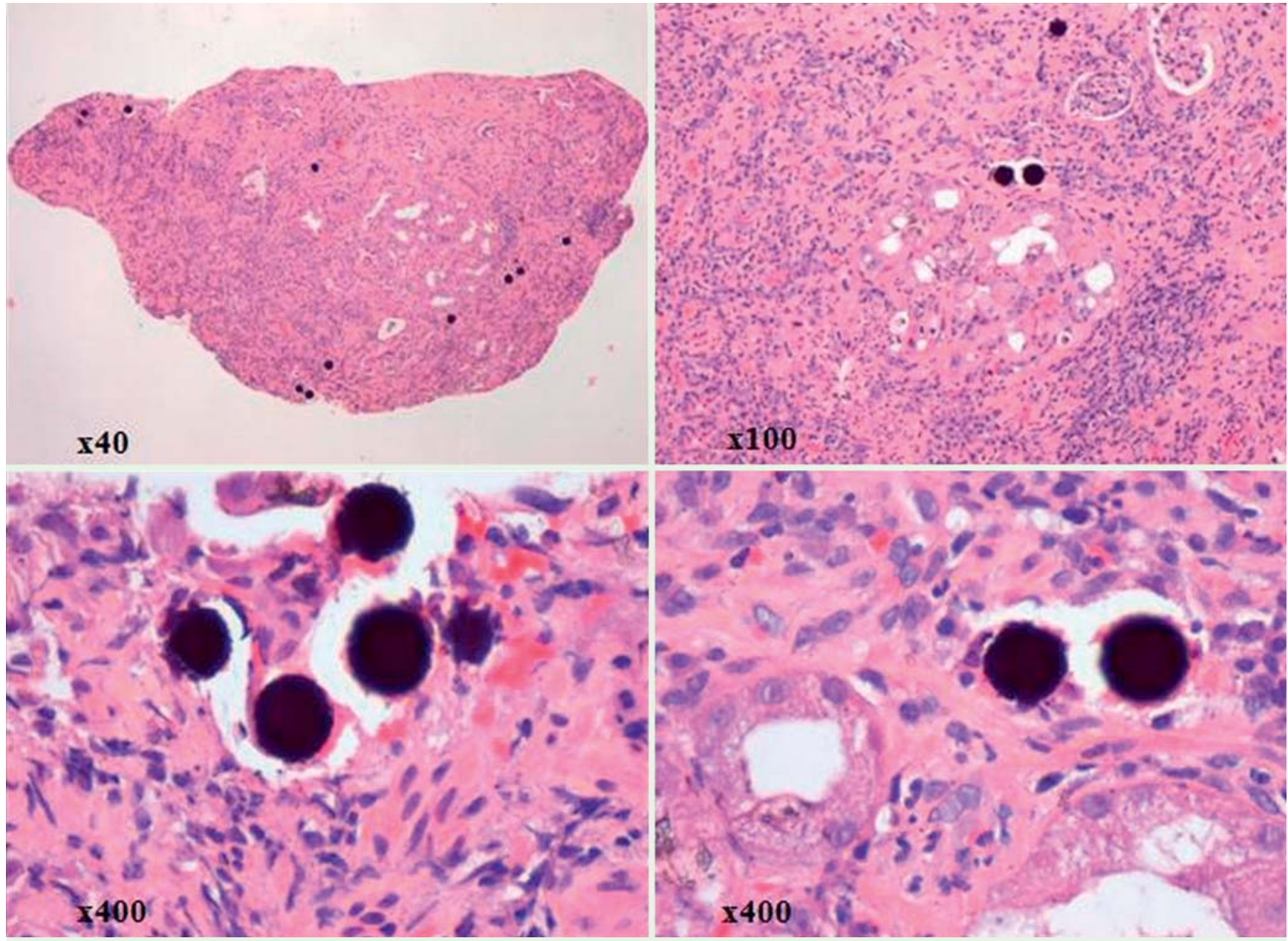

Fig. 4 Histological appearance of the biopsy specimens stained by hematoxylin and eosin (H\&E) showing extensively ulcerated antral gastric mucosa with granulation tissue and the presence of numerous ${ }^{90} \mathrm{Y}$ resin microspheres in the lamina propria. Scattered residual gastric glands showing marked cytological atypia are also present.

ed gastrointestinal tract blood vessels that may sometimes be obscured in liver angiograms despite a detailed pretreatment hepatic angiographic study. The literature reports gastrointestinal complications rates from SIRT of 3\%-24\% [2], with complications resulting from direct radiation toxicity rather than ischemia [3].

A high degree of suspicion is required to make this diagnosis because there are no specific clinical or endoscopic features. Definitive diagnosis is made pathologically by visualization of the pathognomonic microspheres. Treatment with high-dose proton pump inhibitors is recommended, although a response rate under $50 \%$ is to be expected $[2,4]$.

Endoscopy_UCTN_Code_CCL_1AB_2AD_3AC

Competing interests: None

\section{N. Veloso¹ , C. Brandão ${ }^{1}$, B. Gonçalves², L. Costa ${ }^{2}$, N. Coimbra ${ }^{3}$, M. Jacome ${ }^{3}$, L. Moreira Dias ${ }^{1}$}

${ }^{1}$ Department of Gastroenterology, Portuguese Oncology Institute of Porto, Porto, Portugal

2 Department of Interventional Radiology, Portuguese Oncology Institute of Porto, Porto, Portugal

3 Department of Pathology, Portuguese Oncology Institute of Porto, Porto, Portugal

\section{References}

1 Townsend A, Price T, Karapetis C. Selective internal radiation therapy for liver metastases from colorectal cancer. Cochrane Database Syst Rev 2009; 4: CD007045

2 Konda A, Savin MA, Cappell MS et al. Radiation microsphere-induced GI ulcers after selective internal radiation therapy for hepatic tumors: an underrecognized clinical entity. Gastrointest Endosc 2009; 70: 561 - 567

3 Murthy R, Brown DB, Salem $R$ et al. Gastrointestinal complications associated with he- patic arterial Yttrium-90 microsphere therapy. J Vasc Interv Radiol 2007; 18: 553-561

4 Naymagon S, Warner RR, Patel $K$ et al. Gastroduodenal ulceration associated with radioembolization for the treatment of hepatic tumors: an institutional experience and review of the literature. Dig Dis Sci 2010; 55: $2450-2458$

\section{Bibliography}

DOI http://dx.doi.org/

$10.1055 / \mathrm{s}-0032-1326346$

Endoscopy 2013; 45: E108-E109

(c) Georg Thieme Verlag KG

Stuttgart · New York

ISSN 0013-726X

\section{Corresponding author}

\section{N. Veloso, MD}

Department of Gastroenterology Hospital Espírito Santo

Évora

Portugal

Fax: +351-266-740117

nuno_veloso@hotmail.com 